\title{
Is the Turbidimetric Immunoassay of Haptoglobin Phenotype-Dependent?
}

\author{
H. J. M. VAN RIJN, ${ }^{1}$ W. VAN DER WILT, ${ }^{1}$ J. W. STROES, ${ }^{1}$ and J. SCHRIJVER ${ }^{2}$ \\ Clinical and Hematological Laboratory, ${ }^{1}$ Dr. A. Mathijsen Hospital, Utrecht, The Netherlands and \\ Department of Clinical Biochemistry, ${ }^{2}$ TNO-CIVO Toxicology and Nutrition Institute, Zeist, \\ The Netherlands
}

\begin{abstract}
Comparison of the turbidimetric immunossay of haptoglobin with a reference method (the RID technique with appropriate correction for phenotype) clearly showed the turbidimetric assay to be phenotypedependent. Correction factors for the three main phenotypes were calculated and reference values determined.

The observed overall reference range of haptoglobin, irrespective of the phenotype, was $0.56-3.76 \mathrm{~g} / \mathrm{L}$. After correction for phenotype, reference values for the three types were: $\mathrm{Hp} \mathrm{1-1:0.77-2.49;} \mathrm{Hp}$ 2-1:0.86-4.76; and $\mathrm{Hp} 2-2: 0.48-3.15 \mathrm{~g} \mathrm{Hp} / \mathrm{L}$. From our reference values for the several phenotypes of haptoglobin it may be argued that phenotyping is desirable only when the uncorrected haptoglobin value, determined by turbidimetry, is within the range $0.38-0.98 \mathrm{~g} / \mathrm{L}$. Limited to hemolytic diseases, phenotype determination can be omitted beyond this range.
\end{abstract}

KEY WORDS: haptoglobins; immunoturbidimetry; phenotype; reference values.

Tnitially, methods for the determination of haptoIglobin $(\mathrm{Hp})$ were based on the affinity of $\mathrm{Hp}$ binding to hemoglobin. This binding enhances the pseudoperoxidase activity of hemoglobin $(\mathrm{Hb})$ and this property was used in the first methods described for the quantitation of $\mathrm{Hp}$ (1). Many modifications of this enzymatic method, some permitting the use of automatic analyzers, have since been published (2-4). Other methods are based upon the altered spectrophotometric properties of bound hemoglobin $(5,6)$ or upon the separation of the $\mathrm{Hb}-\mathrm{Hp}$ complex from unbound hemoglobin $(2,7,8)$. The results of all these methods are expressed in hemoglobin binding capacity (HBC), i.e., in gram or mol $\mathrm{Hb}$ per litre serum or plasma. No attempts have been made to relate the $\mathrm{HBC}$ to the actual $\mathrm{Hp}$ concentration in $\mathrm{g} / \mathrm{L}$, perhaps wisely so, because this would have revealed a phenotype dependency. The three most common Hp phenotypes, Hp 1-1, Hp 2-1, and Hp 2-2, differ in the $\alpha$ part of the $\alpha \beta$ subunit: Hp 1-1 contains $\alpha_{1} \beta$ subunits only, Hp 2-2 $\alpha_{2} \beta$ only, and Hp 2-1 contains both types. Because $\alpha_{1}$ is smaller than $\alpha_{2}, 1 \mathrm{~g} \mathrm{Hp} 1-1$ contains more $\alpha \beta$ subunits than $1 \mathrm{~g} \mathrm{Hp} \mathrm{2-1}$ or Hp 2-2, and therefore $1 \mathrm{~g} \mathrm{Hp} \mathrm{1-1}$ can bind more hemoglobin. However, since $\mathrm{HBC}$ is measured per $\mathrm{mL}$ serum and not per gram haptoglobin, it is essentially a property of the serum under investigation and independent of the Hp phenotype causing it.

In the 1970 s many laboratories began determining serum haptoglobin levels immunochemically, using the

\footnotetext{
Correspondence: Dr. H. J. M. van Rijn, Dr. A. Mathijsen Hospital, P.O. Box 90.000, 3509 AA Utrecht, The Netherlands.

Manuscript received August 6, 1986; revised December 18, 1986; accepted January 7, 1987.
}

radial immunodiffusion (RID) technique (9). Although it is a very easy technique to use, it has the drawback of diffusion times of up to several days. Recently, immunonephelometric and immunoturbidimetric methods have been developed that require a much shorter time of analysis and allow automatic analyzers to be used $(10-13)$.

The results of immunochemical methods are usually expressed in $\mathrm{g} / \mathrm{L}$, although what is being measured is, in fact, the "anti-Hp binding capacity" of the serum. So, in contrast to methods expressing results in $\mathrm{HBC}$, phenotype dependency can be expected. If the anti-Hp binds equally well to the $\alpha_{1} \beta$ and $\alpha_{2} \beta$ subunits of $\mathrm{Hp}, 1 \mathrm{~g} \mathrm{Hp}$ 1-1 will bind more anti-Hp than $1 \mathrm{~g} \mathrm{Hp} 2-1$ or $\mathrm{Hp} \mathrm{2-2}$ simply because it contains more $\alpha \beta$ subunits. Indeed phenotype dependency has been clearly demonstrated for the RID method (14). If the Hp concentration is read off from a mixed Hp phenotype standard curve, correction factors should be applied to obtain the "real" concentration in $\mathrm{g} \mathrm{Hp} / \mathrm{L}$.

The phenotype dependency of most of the spectrophotometric methods for quantifying the $\mathrm{Hp}$-anti- $\mathrm{Hp}$ complex has not yet become clear. The recent introduction of a Technicon RA-1000 automatic analyzer in our laboratory has given us the opportunity of investigating the phenotype dependency of a turbidimetric method for the determination of haptoglobin. The RID method according to Mancini (9) was selected as the method of reference. Combined with the appropriate correction factors for the three main Hp phenotypes (15), results from this method accurately reflect the real $\mathrm{Hp}$ concentration in $\mathrm{g} \mathrm{Hp} / \mathrm{L}$.

\section{Materials and methods}

\section{INSTRUMENTATION}

Turbidimetric analysis was performed using a Technicon RA-1000 analyzer (Technicon Inc., Tarrytown NY 10591, USA).

\section{REAGENTS}

Anti-haptoglobin antiserum (Cat. No. T21-0455), specific protein sample diluent (Cat. No. T21-1547), specific protein antiserum diluent (Cat. No. T21-1548), and automated immunoprecipitin (AIP) reference serum (Cat. No. T03-0495) were obtained from Technicon. 
Antiserum was diluted 1:20 with specific protein antiserum diluent in accordance with the manufacturer's instructions. After $10 \mathrm{~min}$ the diluted antiserum was filtered through a $22 \mu \mathrm{m}$ filter. AIP reference serum for calibration was diluted using specific protein sample diluent.

For the radial immunodiffusion technique, commercially available immunodiffusion plates (M-partigen) and human protein standard serum B (Cat. No. RDT 02) were obtained from Behringwerke AG (Marburg/Lahn, FRG). The plates contain monospecific polyvalent antiserum, produced by rabbits immunized with purified human haptoglobins.

\section{Methods}

As stated earlier (14), Mancini's method (9) was used for the RID technique. Typing of haptoglobin was done by polyacrylamide gel electrophoresis (14). Initial results were obtained from a mixed haptoglobin-type standard curve and subsequently corrected for phenotype. The correction factors used were $0.6,1.3$, and 1.5 for phenotypes $1-1,2-1$, and $2-2$, respectively, in accordance with the information supplied by the manufacturer of the RID plates.

The haptoglobin determination on the Technicon RA-1000 can be summarized as follows: the immunological complex formed between haptoglobin and the haptoglobin antibody is measured turbidimetrically at $340 \mathrm{~nm}$ after 5 min incubation.

Polyethylene glycol in the reaction mixture accelerates the formation of the complex. A standard curve for the RA-1000 was generated with the AIP reference serum and five dilutions of it. The AIP reference serum was assigned a value of $5.06 \mathrm{~g} / \mathrm{L}$ determined using RID, our chosen method of reference. Unknown values are automatically calculated by reading absorbances from the standard curve. Patient samples with haptoglobin values higher than the highest value of the standard curve $(5.06 \mathrm{~g} / \mathrm{L})$ were diluted.

\section{STATISTICAL METHODS}

The coefficient of variation (CV) of the results of duplicate determinations was estimated by the formulas described by Abraham et al. (16). Linear regression equations were presented with zero intercept if this did not significantly increase the variance. This was tested by comparison of the regression line with intercept $a$ and the regression line with zero intercept by $\mathrm{F}$ test at the $10 \%$ probability level (17).

\section{Results}

\section{LINEARITY}

To determine the linearity of the turbidimetric method aliquots of sera with different phenotypes were diluted with specific protein sample diluent. The turbidimetric method appeared to be linear up to $5.06 \mathrm{~g} / \mathrm{L}$, which adequately spans the range of concentrations encountered in the majority of normal human samples.
TABLE 1

Precision Studies

\begin{tabular}{lll}
\hline & \multicolumn{1}{c}{ Within-assay } & Between-assay \\
\hline Range $(\mathrm{g} / \mathrm{L})$ & $0.48-(n=15)$ & $0.46-1.65(n=15)$ \\
Mean $(\mathrm{g} / \mathrm{L})$ & 1.19 & 1.13 \\
SD $(\mathrm{g} / \mathrm{L})$ & 0.04 & 0.11 \\
CV $(\%)$ & 3.1 & 10.2 \\
Range $(\mathrm{g} / \mathrm{L})$ & $2.72-5.05(n=10)$ & $2.72-5.12(n=10)$ \\
Mean $(\mathrm{g} / \mathrm{L})$ & 4.00 & 3.96 \\
SD $(\mathrm{g} / \mathrm{L})$ & 0.09 & 0.20 \\
CV $(\%)$ & 2.3 & 5.1 \\
\hline
\end{tabular}

Within-assay studies and between-assay studies were performed with patient samples without separation to phenotype. Results are presented as the uncorrected values from turbidimetric analysis. SD and CV represent the standard deviation and coefficient of variation and between the duplicates

\section{Precision}

Precision was studied by "within-run" and "betweenrun" sample analysis. For both methods, low-content and high-content samples were examined and the precision was expressed in terms of the coefficient of variation (CV). The "within-assay" variance was evaluated from a series of duplicate measurements of samples randomly distributed in a single assay. The "betweenassay" variance was calculated from samples analysed in two different assays using anti-haptoglobin antisera with different lot numbers. Within- and between-assay precision of the turbidimetric method at two concentrations have been summarized in Table 1 . The results indicate that the precision obtained is adequate, being approximately the same as that obtained with other immunochemical methods (13).

\section{Phenotype dependency}

Results obtained with our reference method (RID corrected for phenotype) expressed in $\mathrm{g} \mathrm{Hp} / \mathrm{L}$ (taken as $x$ ) were compared with those of the turbidimetric method (taken as $y$ ). For 74 routinely obtained samples, linear regression yielded the following equation: $y=0.85$ $( \pm 0.04) x, r=0.93$. (F test $a \neq 0$ vs $a=0 \rightarrow 1.27 \mathrm{~ns}$ ).

The following data also show that the three phenotypes do not behave identically. The regression equation for samples of the haptoglobin phenotype $1-1$ was $y=1.27( \pm 0.09) x, r=0.97, n=14$ (F test $a=0$ vs $a \neq 0$ $\rightarrow 1.31 \mathrm{~ns})$; for samples of the haptoglobin phenotype $2-1, y=0.79( \pm 0.03) x, r=0.97, n=42$ (F test $a=0$ vs $a \neq 0 \rightarrow 1.05 \mathrm{~ns})$; and for the samples of the haptoglobin phenotype $2-2, y=0.80( \pm 0.06) x, r=0.96, n=18$ (F test $a=0$ vs $a \neq 0 \rightarrow 1.27 \mathrm{~ns}$ ).

So, if the results from the turbidimetric analysis are expressed in $\mathrm{g} \mathrm{Hp} / \mathrm{L}$ a clear phenotype dependency can be observed. A specific conversion factor for each $\mathrm{Hp}$ phenotype has to be introduced to obtain the "true" $\mathrm{Hp}$ concentration (i.e., the value obtained by our method of reference in $\mathrm{g} \mathrm{Hp} / \mathrm{L})$. These factors are $0.79(1 / 1.27)$, $1.27(1 / 0.79)$, and $1.25(1 / 0.80)$ for Hp 1-1, Hp 2-1, and Hp 2-2, respectively. 


\section{EFFECT OF PHENOTYPE ON Hp IMMUNOASSAY}

TABLE 2

Reference Values for Haptoglobin Expressed in g $\mathrm{Hp} / \mathrm{L}$

\begin{tabular}{crrrr}
\hline Type & $N$ & $P 50$ & $P 2.5$ & $P 97.5$ \\
\hline Overall & 231 & 1.62 & 0.56 & 3.76 \\
$1-1$ & 45 & 1.56 & 0.77 & 2.49 \\
$2-1$ & 119 & 2.17 & 0.86 & 4.76 \\
$2-2$ & 67 & 1.35 & 0.48 & 3.15 \\
\hline
\end{tabular}

Based on turbidimetric analysis. Results for the specific phenotypes were corrected with the appropriate factors $(0.79$, 1.27 , and 1.25 for $\mathrm{Hp} 1-1, \mathrm{Hp} 2-1$, and $\mathrm{Hp} 2-2$, respectively.

\section{REFERENCE RANGE}

An overall reference range has been determined using samples collected from regular blood donors (men and women ages 18-62). A total of 231 samples were assayed. As the haptoglobin concentrations were not normally distributed, reference values were determined using the distribution-free method described by Rümke and Bezemer (18). The limits of percentiles were set at $2.5 \%$ and $97.5 \%$ with a reliability of $95 \%$. The $P 2.5, P 50$, and $P 97.5$ of the turbidimetrically measured haptoglobin concentrations of this population were $0.56,1.62$, and $3.76 \mathrm{~g} / \mathrm{L}$, respectively. As can be seen from Table 2 , the reference values for the three different types of $\mathrm{Hp}$ clearly differed. No differences were observed between samples from men and women with the same phenotype.

\section{Conclusions}

Determining of the phenotype dependency of methods for measuring serum haptoglobin is extremely important. With the RID method, phenotyping is often necessary to decide what is "below normal," "normal," or "above normal". A value read from a mixed type standard curve has to be multiplied by 1.5 (with Hp 2-2) or 0.6 (with $\mathrm{Hp} \mathrm{1-1)} \mathrm{to} \mathrm{obtain} \mathrm{the} \mathrm{"true} \mathrm{Hp"} \mathrm{concentration}$ in $\mathrm{g} \mathrm{Hp} / \mathrm{L}$, obviously a difference of potential clinical significance. Phenotype dependency can also be expected in other immunochemical methods expressing the haptoglobin concentration in $\mathrm{g} \mathrm{Hp} / \mathrm{L}$.

We opted for the RID technique with the appropriate correction factors, as determined with the purified Hp phenotypes (15), as our method of reference because there is no internationally accepted reference preparation or reference method for haptoglobin. It clearly appears from the different regression equations for the three phenotypes obtained in our study that the turbidimetric assay of haptoglobin is phenotype-dependent. A possible advantage over the RID technique is that the difference between the calculated correction factors for the three phenotypes is smaller than with the RID technique. This may result in a smaller interval where the decision on "normal" or "lowered" would depend on the phenotype of the sample under investigation.

Existing literature about the phenotype dependency of the several methods is often confusing, if not contradictory. A crucial point in this respect is the difference in molecular weight between the $\alpha_{1}$ and $\alpha_{2}$ chains: $1 \mathrm{~g} \mathrm{Hp}$
$1-1$ contains more $\alpha \beta$ subunits than 1 g Hp $2-1$ or $\mathrm{Hp}$ $2-2$. If one $\mathrm{Hp} \alpha \beta$ subunit binds one $\mathrm{Hb} \alpha \beta$ subunit or one anti-Hp molecule, $1 \mathrm{~g} \mathrm{Hp} \mathrm{1-1} \mathrm{will} \mathrm{bind} \mathrm{more} \mathrm{Hb}$ or anti-Hp than $1 \mathrm{~g}$ of one of the other phenotypes. Although factors like the degree of polymerization, diffusion rate, and steric hindrance may also be at play, this assumption is essentially true. The absence of polymerization with $\mathrm{Hp}$ 1-1 even tends to enhance the difference between the three phenotypes.

Comparison between methods measuring the $\mathrm{Hb}$ binding capacity and immunochemical methods using turbidimetry (10) or nephelometry (13) yields an excellent correlation, independent of the phenotype. From these studies a linear relation can be concluded to exist between the hemoglobin binding capacity and the "antihaptoglobin binding capacity" of the serum. It is important to note that the $\mathrm{Hp}$ concentration determined in this way cannot be expressed in $\mathrm{g} \mathrm{Hp} / \mathrm{L}(13)$, since the relation between $\mathrm{HBC}$ and $\mathrm{g} H \mathrm{p} / \mathrm{L}$ is different for each phenotype. If one of the two methods truly measures $g$ $\mathrm{Hp} / \mathrm{L}$, phenotype dependency should be observed, as was proven by comparing a kinetic method for measuring $\mathrm{Hb}$ binding capacity with the RID technique (phenotype corrected with the appropriate factors) (3). All methods currently in use measure the $\mathrm{Hb}$ binding capacity or the anti-Hp binding capacity of the serum. If the haptoglobin concentration is represented as the HBC advantageously, no phenotype dependency will be observed. This option is also available with immunochemical methods, since $\mathrm{HBC}$ and anti-Hp binding capacity are apparently analogous concepts. Which is the clinically most relevant measure, $\mathrm{HBC}$ or $\mathrm{g} \mathrm{Hp} / \mathrm{L}$, is as yet unclear. Interestingly, normal values for the three phenotypes differ significantly, whether they are expressed as $\operatorname{HBC}(7,10)$ or as $\mathrm{g} \mathrm{Hp} / \mathrm{L}(14)$. So, whichever method is chosen, phenotyping may still be necessary within a certain range.

In our laboratory the average normal values of the three phenotypes $1-1,2-1$, and $2-2$ as determined by the turbidimetric assay were $1.56(0.77-2.49), 2.17$ $(0.86-4.76)$, and $1.35(0.48-3.15) \mathrm{g} \mathrm{Hp} / \mathrm{L}$, respectively, after correction for phenotype. These values closely resemble those determined with the RID method (14): $1.40(0.70-2.30), 2.10(0.90-3.60)$, and $1.65(0.60-2.90)$ g Hp/L, respectively.

In this context the question arises whether it is always necessary to perform a haptoglobin phenotyping. Based on results obtained earlier in our laboratory $(14,19,20)$ and the results obtained in this study, we advocate that phenotyping be performed only when indispensable for clinical purposes, i.e., when the uncorrected $H p$ value obtained from the RA-1000 is within the range $0.38-0.98 \mathrm{~g} / \mathrm{L}$. In this range the decision as regards lowered or normal depends on the phenotype. An uncorrected $\mathrm{Hp}$-value of $0.38 \mathrm{~g} / \mathrm{L}$ would be corrected to $0.30,0.48$, or 0.48 depending on the phenotype being $\mathrm{Hp} \mathrm{1-1,} \mathrm{Hp} \mathrm{2-1,} \mathrm{or} \mathrm{Hp} \mathrm{2-2,} \mathrm{respectively,} \mathrm{which} \mathrm{is} \mathrm{in} \mathrm{all}$ cases below the normal range, and an uncorrected value of $0.98 \mathrm{~g} / \mathrm{L}$ would be corrected to $0.77,1.24$, or 1.23 , respectively, which is within the normal range in all cases. When the level is above normal, indicating for instance an acute inflammatory process, phenotyping 


\section{VAN RIJN, VAN DER WILT, STROES, AND SCHRIJVER}

can be omitted. Also, in the case of a strongly hemolytic process with values below $0.38 \mathrm{~g} / \mathrm{L}$, determination of the genetic type will not influence the conclusion.

Performing assays with a Technicon RA-1000 analyzer has several advantages over using the RID method. Its high throughput of 50 specimens per hour is very much in excess of what is possible with the RID method. In conclusion, the method was found to be rapid (time of analysis is only $6 \mathrm{~min}$ ) and to provide acceptable precision. Only in a few cases is the advantage of speed of analysis lost by need for phenotyping.

\section{IReferences}

1. Jayle MF. Methode de dosage de l'haptoglobin serique. Bull Soc Chim Biol 1951; 33: 876-80.

2. Pintera J. The biochemical, genetic, and clinicopathological aspects of haptoglobin. Ser Haem 1971; 42: 7-183.

3. Standing S, Price CP. A kinetic method for the determination of haptoglobin as haemoglobin binding capacity. Clin Chim Acta 1976; 66: 393-403.

4. Kickler TS, Fong PF, Johnson GF, Solomom HM. Kinetic determination of serum haptoglobin with a centrifugal analyser. Clin Chem 1976; 22: 1962-7.

5. Calhoun DB, Englander SW. Measurement of the haptoglobin concentration in plasma and other fluids by a simple spectrometric procedure. Anal Biochem 1979; 99: $421-6$.

6. Shim BS, Jue DM. Simple determination of haptoglobin level in serum. Clin Chim Acta 1984; 136: 145-53.

7. Nijman M. Serum haptoglobin. Methodological and clinical studies. Scand J Clin Lab Invest 195911 Suppl. 39: $1-169$.

8. Javid J. Human haptoglobins. Curr Topics Hematol 1978; 1: $151-92$.
9. Mancini G, Carbonara AU, Heremans JF. Immunochemical quantitation of antigens by single radial immunodiffision. Immunochemistry 1965; 2: 235-54.

10. Ramakers JM, Kreutzer HJH. Turbidimetric determination of haptoglobin. $J$ Clin Chem Clin Biochem 1976; 14: 407-10.

11. Sternberg JC. A rate nephelometer for measuring specific proteins by immunoprecipitin reactions. Clin Chem 1977; 23: $1456-64$

12. Moseley MJ. An immunoturbidimetric method for the estimation of human haptoglobin using centrifugal analysis. Clin Chim Acta 1980; 102: 153-60.

13. Van Lente F, Marchand A, Galen RS. Evaluation of a nephelometric assay for haptoglobin and its clinical usefulness. Clin Chem 1979; 25: 2007-10.

14. Schrijver J, van Rijn HJM, Schreurs WHP. Reevaluation of the haptoglobin reference values with the radial immunodiffusion technique. Clin Biochem 1984; 17: 258-62.

15. Braun HJ, Aly FW. Problems in the quantitative estimation of human serum haptoglobin by single radial immunodiffusion. Clin Chim Acta 1969; 26: 588-90.

16. Abraham GE, Swerdhof R, Tulchinsky D. Radioimmunassay of plasma progesterone. $J$ Clin Endocrinol 1971; 32: 619-24.

17. Guiguet M, Padieu P, Mack G. Laser nephelometric measurement of seven serum proteins compared with radial immunodiffusion. I Clin Chem Clin Biochem 1983; 21: 217-21.

18. Rumke Chr L, Bezemer PD. Methods for determinations of normal values. II. New methods. Ned T Geneesk 1972; 116: $1559-68$.

19. van Rijn HJM, Kruit WHJ, Schrijver J. Haptoglobin typing, is it clinically necessary for a reliable determination of haptoglobin with the single radial immunodiffusion technique. J Clin Chem Clin Biochem 1984; 22: 109-12.

20. van Rijn HJM, Sanderink GJCM. Haptoglobin a useful laboratory parameter? Neth J Med 1986; 29: 16-22. 